\title{
The Expected Value of a Fuzzy Number
}

\author{
Mohamed Shenify, Fokrul Alom Mazarbhuiya \\ College of Computer Science, Albaha University, Albaha, Saudi Arabia \\ Email: mshenify@yahoo.com, fokrul2005@yahoo.com
}

Received 12 October 2014; revised 13 November 2014; accepted 29 November 2014

Academic Editor: Zhongzhi Shi, Institute of Computing Technology, CAS, China

Copyright (C) 2015 by authors and Scientific Research Publishing Inc.

This work is licensed under the Creative Commons Attribution International License (CC BY). http://creativecommons.org/licenses/by/4.0/

(c) (i) Open Access

\begin{abstract}
Conjunction of two probability laws can give rise to a possibility law. Using two probability densities over two disjoint ranges, we can define the fuzzy mean of a fuzzy variable with the help of means two random variables in two disjoint spaces.
\end{abstract}

\section{Keywords}

Probability Density Function, Probability Distribution, Fuzzy Measure, Fuzzy Expected Value, Fuzzy Mean, Fuzzy Membership Function, Dubois-Prade Reference Functions

\section{Introduction}

Zadeh [1] introduced the concept of fuzziness into the realm of mathematics. Accordingly, various authors have studied the mathematics related to the fuzzy measure and the associated fuzzy expected value [2]-[7] studied the fuzzy expected value and its associated results by defining the fuzzy expected value in terms of fuzzy measure. In their definition they tried to find the fuzzy expected value of a possibility distribution. In [8], authors developed a new method of analysis of possibilistic portfolio that associates a probabilistic portfolio. Similar works were done in associating possibility and probability [9] [10]. In [11] [12], the author tries to establish a link between possibility law and probability law using a concept discussed in the paper called set superimposition [13]. In [14], the author tries to establish a link between and randomness.

In this article, using the superimposition of sets, we have attempted to define the expected value of a fuzzy variable in term of expected values of two random variables in two disjoint spaces. It can be seen that the expected value of a fuzzy number is again a fuzzy set.

\section{Definitions and Notations}

Let $X$ be a continuos random variable in the interval $[a, b]$ with probability density function $f(x)$ and 
probability distribution function $F(x)$. Then

$$
\operatorname{Prob}(a \leq X \leq b)=\int_{a}^{b} f(y) \mathrm{d} y=F(b)-F(a)
$$

Further, the expected value of $X$ would be

$$
E(X)=\int_{-\infty}^{\infty} x \mathrm{~d} F(x)
$$

where the integral is absolutely convergent.

Let $E$ be a set and $x \in X$ then we can define a fuzzy subset $A$ of $E$ as

$$
A=\left\{x, \chi_{A}(x) ; x \in E\right\}
$$

where $\chi_{A}(x) \in[0,1]$ is the fuzzy membership function of the fuzzy set $A$ for an ordinary set, $\chi_{A}(x)=0$ or 1 . A fuzzy set $A$ is called normal if $\chi_{A}(x)=1$ for at least one $x \in E$.

A $\alpha$-cut $A_{\alpha}$ for a fuzzy set $A$ is an ordinary set of elements such that $\chi_{A}(x) \geq \alpha$ for $0 \leq \alpha \leq 1$, i.e. $A_{\alpha}=\left\{x \in E, \chi_{A}(x) \geq \alpha\right\}$.

The membership function of a fuzzy set is known as a possibility distribution [15]. We usually denote a fuzzy number by a triad $[a, b, c]$ such that $\chi_{A}(a)=0=\chi_{A}(c)$ and $\chi_{A}(b)=1 . \chi_{A}(x)$, for $x \in[a, b]$, is the left reference function and for $x \in[b, c]$ is the right reference function. The left reference function is right continuous, monotone and non-decreasing, while the right reference function is left continuous, monotone and nonincreasing. The above definition of a fuzzy number is known as an L-R fuzzy number.

\section{Kandel's Definition of a Fuzzy Measure}

Kandel [5] [16] has defined a fuzzy measure as follows: Let $B$ be a Borel field ( $\sigma$-algebra) of subset of the real line $\Omega$. A set function $\mu($.$) defined on B$ is called fuzzy measure if it has the following properties:

(1) $\mu(\phi)=0 \quad(\phi$ is the empty set);

(2) $\mu(\Omega)=1$;

(3) If $\mathrm{A}, \mathrm{B} \in \mathrm{B}$ with $\mathrm{A} \subset \mathrm{B}$, then $\mu(\mathrm{A}) \leq \mu(\mathrm{B})$;

(4) If $\left\{\mathrm{A}_{i} ; 1 \leq i \leq \infty\right\}$ is a monotonic sequence, then $\underset{i \rightarrow \infty}{\operatorname{Lim}}\left[\mu\left(\mathrm{A}_{i}\right)\right]=\mu\left[\operatorname{Lim}_{i \rightarrow \infty}\left(\mathrm{A}_{i}\right)\right]$ Clearly, $\phi, \Omega \in B$. Also, if $\mathrm{A}_{i} \in B$, then $\operatorname{Lim}_{i \rightarrow \infty}\left(\mathrm{A}_{i}\right) \in B .(\Omega, B, \mu)$ is called a fuzzy measure space. $\mu($.$) is the fuzzy measure of$ $(\Omega, B)$.

Let $\chi_{A}: \Omega \rightarrow[0,1]$ and $A_{\alpha}=\left\{x ; \chi_{A}(x) \geq \alpha\right\}$. The function $\chi_{A}$ is called a $B$-measurable function, if $A_{\alpha} \in B$ for all $\alpha \in[0,1]$. In their notations, fuzzy expected value is defined as follows: Let $\chi_{A}$ be a $B$ measurable function such that $\chi_{A} \in[0,1]$. The fuzzy expected value (FEV) of $\chi_{A}$ over a set $A$ with respect to the measure $\mu($.$) is defined as \operatorname{Sup}_{\alpha \in[0,1]}\left\{\min \left[\alpha . \mu\left(A_{\alpha}\right)\right]\right\}$.

Now $\mu\left\{x ; \chi_{A}(x.) \geq \alpha\right\}=f_{A}(\alpha)$ is a function of the threshold $\alpha$. The calculation of FEV $\left(\chi_{A}\right)$ then consists of finding the intersection of the curves of $\alpha=f_{A}(\alpha)$. The intersection of the curves will be at a value $\alpha=H$ so that FEV $\left(\chi_{A}\right)=H \in[0,1]$ as in the diagram.

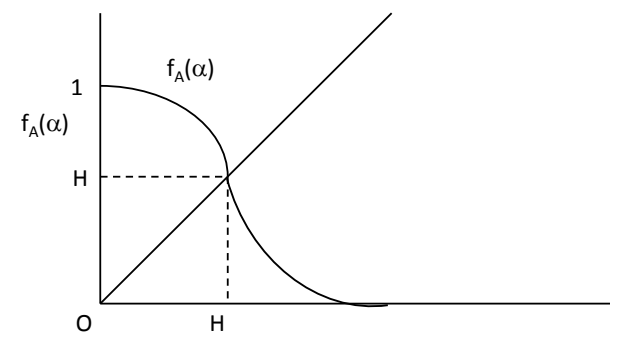




\section{Definition of an Expected Value of Fuzzy Number}

Kandel's definition of a fuzzy expected value is based on the definition of the fuzzy measure. However, the fuzzy measure being non-additive is not really a measure.

Baruah [13] has shown that instead of expressing a fuzzy measure in $[a, b, c]$, if we express the possibility distribution first as a probability distribution function in $[a, b]$ and then as a complementary probability distribution function in $[b, c]$, the mathematics can be seen to be governed by the product measure on $[a, b]$ and $[b, c]$. As such, the question of non-additivity of the fuzzy measure does not come into picture.

We propose to define the fuzzy expected value or the possibilistic mean based on the idea that two probability measures can give rise to a possibility distribution. In other words, the concerned possibilistic measure need not be fuzzy at all.

Accordingly, we propose to define a possibilistic mean as follows: Let $X$ be a fuzzy variable in the fuzzy set $A=[a, b, c]$. We divide $A$ into two intervals $A_{1}=[a, b]$ and $A_{2}=[b, c]$ such that $A_{1} \cup A_{2}=A$ and $A_{1} \cap A_{2}=\phi$. Let $X$ be a random variable on $A_{1}$. Then from (1), the mean of $X$ would be

$$
E_{1}(X)=\int_{a}^{b} x f(x) \mathrm{d} x
$$

where $f(x)$ is the concerned probability density function defined on $[a, b]$. Let the mean of the random variable an $A_{2}$ be

$$
E_{2}(X)=\int_{b}^{c} x g(x) \mathrm{d} x
$$

where $g(x)$ is the concerned probability density function defined on $[b, c]$.

Thus, from (2) and (3), we get the possibilistic mean of $X \in[a, b, c]$ as

$$
M=\left[\int_{a}^{b} x f(x) \mathrm{d} x, b, \int_{b}^{c} x g(x) \mathrm{d} x\right]=\left\{x, \chi_{M}(x) \in[r, 1], x \in E\right\}
$$

where $r=\min \left\{\chi_{M}\left(\int_{a}^{b} x f(x) \mathrm{d} x\right), \chi_{M}\left(\int_{b}^{c} x g(x) \mathrm{d} x\right)\right\}$.

Equation (4) is our required result that shows that poissibilistic mean of a fuzzy variable is again a fuzzy set.

To illustrate the result (4), we take $A=[a, b, c]$, a triangular number such that $\chi_{A}(a)=0=\chi_{A}(c)$ and $\chi_{A}(b)=1$. The probability distribution function is given by

$$
F(x)=\left[\begin{array}{ll}
0 & x \leq a \\
\frac{(x-a)}{(b-a)} & a \prec x \prec b \\
1 & x \geq b
\end{array}\right.
$$

where

$$
f(x)=\frac{1}{(b-a)}
$$

is the probability density function in $a \leq x \leq b$.

The complementary probability distribution or the survival function is given by

$$
G(x)=\left[\begin{array}{ll}
1 & x \leq b \\
1-\frac{(x-b)}{(c-b)} & b<x<c \\
0 & x \geq c
\end{array}\right.
$$

where $F(x)=1-G(x)$ and the probability density function in $b \leq x \leq c$ is 


$$
g(x)=\frac{1}{(c-b)}
$$

Therefore, the expected value of a uniform random variable $X$ on $[a, b]$ is

$$
E_{1}(x)=\frac{(a+b)}{2}
$$

and similarly, the expected value of another uniform random variable $X$ on $[b, c]$ is

$$
E_{2}(x)=\frac{(b+c)}{2}
$$

Equations (9) and (10) together give the expected value of a triangular fuzzy variable in $[a, b, c]$ as

$$
M^{\prime}=\left[\frac{(a+b)}{2}, b, \frac{(b+c)}{2}\right]=\left\{x, \chi_{M^{\prime}}(x) \in[p .1], x \in E\right\}
$$

where $p=\min \left\{\chi_{M^{\prime}}\left(\frac{(a+b)}{2}\right), \chi_{M^{\prime}}\left(\frac{(b+c)}{2}\right)\right\}=1 / 2$.

Equations (4) and (11) show that the expected value of a fuzzy number is again a fuzzy set.

\section{Conclusion}

The very definition of a fuzzy expected value as given by Kandel is based on the understanding that the so called fuzzy measure is not really a measure in the strict sense. The possibility distribution function is viewed as two reference functions. Using left reference function as probability distribution function and right reference function as survival function, in this article we redefine the expected value of a fuzzy number which is again a fuzzy set.

\section{References}

[1] Zadeh, L.A. (1965) Fuzzy Sets as Basis of Theory of Possibility. Fuzzy Sets and Systems, 1, 3-28. http://dx.doi.org/10.1016/0165-0114(78)90029-5

[2] Aczel, M.J. and Ptanzagl, J. (1966) Remarks on the Measurement of Subjective Probability and Information. Metrica, 5, 91-105.

[3] Asai, K., Tanaka, K. and Okuda, T. (1977) On the Discrimination of Fuzzy States in Probability Space. Kybernetes, 6, 185-192. http://dx.doi.org/10.1108/eb005451

[4] Baldwin, J.F. and Pilsworth, B.W. (1979) Fuzzy Truth Definition of Possibility Measure for Decision Classification. International Journal of Man-Machine Studies, 11, 447-463.

[5] Kandel, A. (1979) On Fuzzy Statistics. In: Gupta, M.M., Ragade, R.K. and Yager, R.R., Eds., Advances in Fuzzy Set Theory and Application, North Holland, Amsterdam.

[6] Kandel, A. and Byatt, W.J. (1978) Fuzzy Sets, Fuzzy Algebra and Fuzzy Statistics. Proceedings of the IEEE 66, USA, January 1978, 1619-1639.

[7] Teran, P. (2014) Law of Large Numbers for Possibilistic Mean Value. Fuzzy Sets and Systems, 245, 116-124. http://dx.doi.org/10.1016/j.fss.2013.10.011

[8] Georgescu, I. and Kinnunen, J. (2011) Credibility Measures in Portfolio Analysis: From Possibilistic to Probabilistic Models. Journal of Applied Operational Research, 3, 91-102.

[9] Sam, P. and Chakraborty, S. (2013) The Possibilistic Safety Assessment of Hybrid Uncertain Systems. International Journal of Reliability, Quality and Safety Engineering, 20, 191-197.

[10] Zaman, K., Rangavajhala, S., Mc Donald, M. and Mahadevan, S. (2011) A Probabilistic Approach for Representation of Interval Uncertainty. Reliability Engineering and System Safety, 96, 117-130. http://dx.doi.org/10.1016/j.ress.2010.07.012

[11] Baruah, H.K. (2010) The Randomness-Fuzziness Consistency Principle. International Journal of Energy, Information and Communications, 1, 37-48.

[12] Baruah, H.K. (2012) An Introduction to the Theory of Imprecise Sets: The Mathematics of Partial Presence. Journal of 
Mathematical and Computational Science, 2, 110-124.

[13] Baruah, H.K. (1999) Set Superimposition and Its Application to the Theory of Fuzzy Sets. Journal of Assam Science Society, 40, 25-31.

[14] Mazarbhuiya, F.A. (2014) Finding a Link between Randomness and Fuzziness. Applied Mathematics, 5, 1369-1374.

[15] Prade, H. (1983) Fuzzy Programming Why and How? Some Hints and Examples. In: Wang, P.P., Ed., Advances in Fuzzy Sets, Possibility Theory and Applications, Plenum Press, New York, 237-251. http://dx.doi.org/10.1007/978-1-4613-3754-6_16

[16] Kandel, A. (1982) Fuzzy Techniques in Pattern Recognition. Wiley Interscience Publication, New York. 
Scientific Research Publishing (SCIRP) is one of the largest Open Access journal publishers. It is currently publishing more than 200 open access, online, peer-reviewed journals covering a wide range of academic disciplines. SCIRP serves the worldwide academic communities and contributes to the progress and application of science with its publication.

Other selected journals from SCIRP are listed as below. Submit your manuscript to us via either submit@scirp.org or Online Submission Portal.
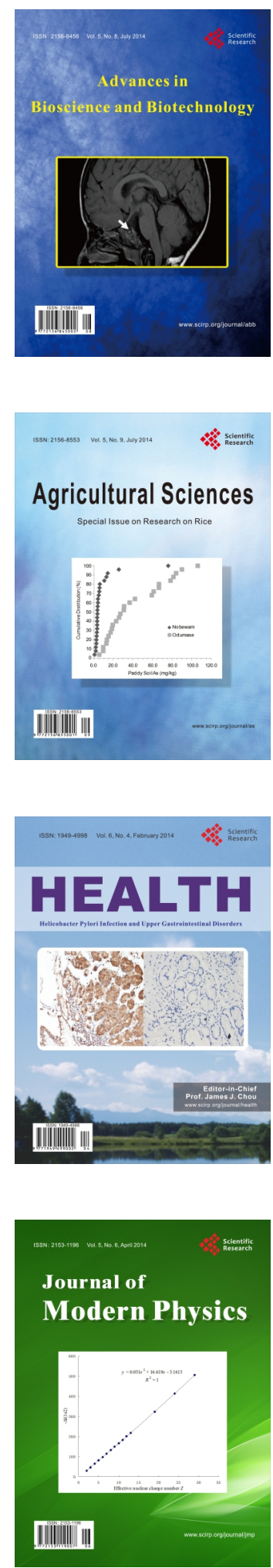
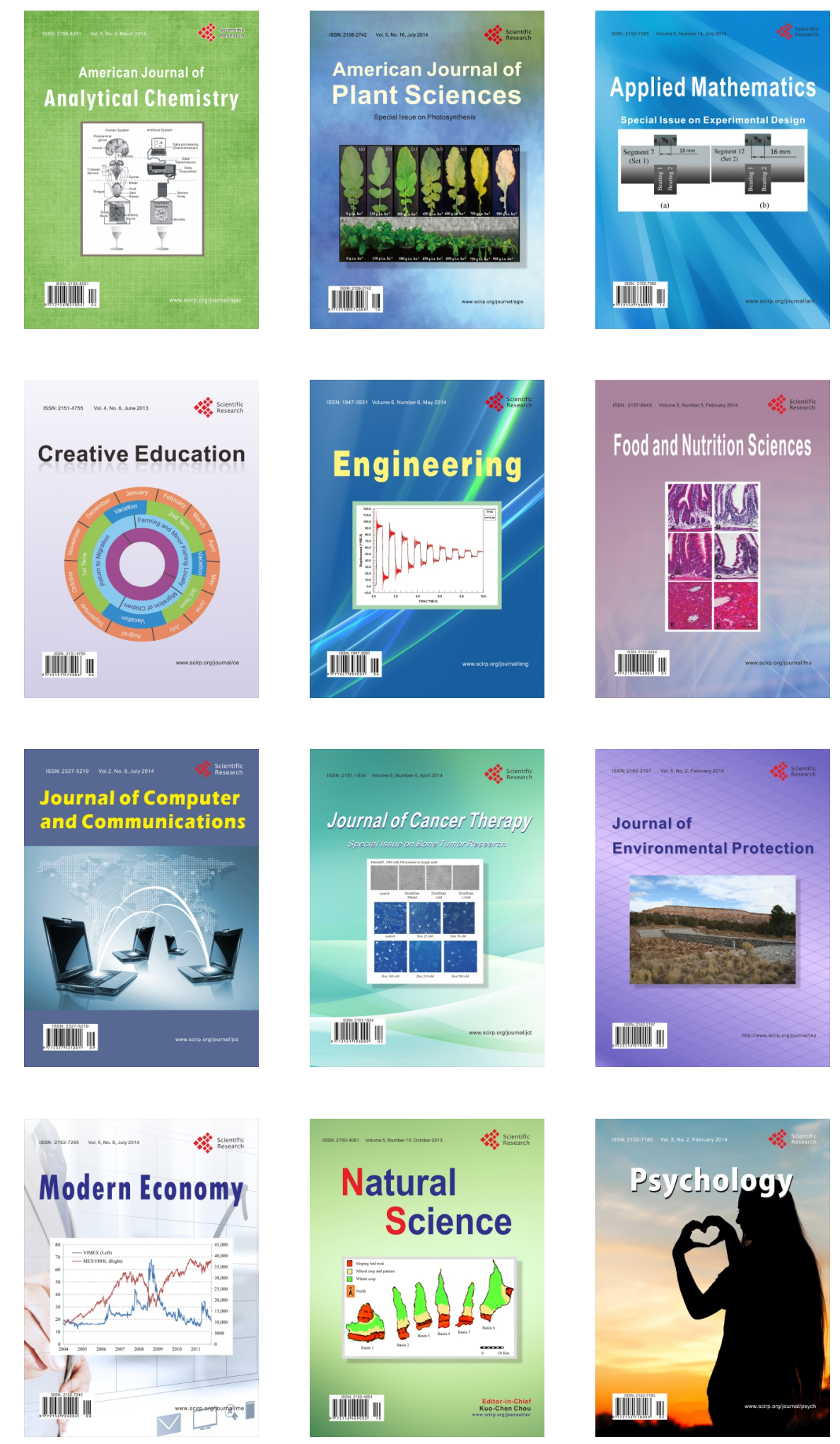